\title{
RELATIVITY AND IRREVERSIBILITY
}

\author{
I. ANTONIOU, E. KARPOV, I. PRIGOGINE, AND G. PRONKO
}

Received 15 December 2003

The Lorentz transformation is extended to the decaying states in a relativistic model of interacting fields. The nonlocal action is defined beyond the Hilbert space. This shows that irreversible extensions of dynamics of Poincaré nonintegrable systems are compatible with relativity.

\section{Introduction}

The dynamical laws of physics in their conventional formulation are time reversible. The description of irreversible phenomena was always thought to be incompatible with the dynamical laws. We have recently discussed $[1,2,3,9,10,11,12]$ the origin of irreversible behaviour due to resonances in various Poincaré nonintegrable quantum systems. The analytic extension of the Hamiltonian or Liouvillian in such systems leads to new solutions describing unstable states, which evolve irreversibly. Such states defined in the wave function space are usually called Gamow vectors $[4,6]$. Thus it was shown that the presence of resonance leads to intrinsic irreversibility of dynamical systems. Therefore, irreversibility is compatible with dynamics.

The aim of the present paper is to show that the irreversible description is compatible with relativistic invariance as well. To consider this question clear is very important, in view of recent publications on this topic, for example, [8].

In order to demonstrate this compatibility, we study the relativistic transformation of decaying unstable Gamow modes using a relativistic invariant model of two interacting fields introduced in [2]. We suggest that the transformation of the Gamow states is induced by the Lorentz transformation of field modes which are eigenstates of the fourdimensional energy momentum of the system. The formal application of the Lorentz boost to the Gamow state leads to a complex value of the momentum $\mathbf{k}^{\prime}$ after the transformation [2]. However, we can keep real the transformed momentum if we consider this transformation at the level of wave packets. Our arguments are based on the fact that the Gamow state $\left|\Psi^{\mathrm{G}}(\mathbf{k})\right\rangle$ with momentum $\mathbf{k}$ is obtained as pole contribution from 
the incoming eigenstate of the energy momentum $\left|\Psi_{\text {in }}(E, \mathbf{k})\right\rangle$ using analytic continuation to the complex plane of the energy $E$ for fixed momentum $\mathbf{k}$. Wave packets observed experimentally in scattering experiments are superpositions of the energy-momentum eigenstates $\left|\Psi_{\text {in }}(E, \mathbf{k})\right\rangle$. The analytic extension to the complex energy plane isolates the contribution of the exponentially decaying Gamow modes to the wave packet. All these Gamow modes have real momenta. The same wave packets will be seen by some moving observer as transformed by proper Lorentz boost. The corresponding analytic extension for the transformed wave packet will isolate other Gamow modes with again real momenta, thus determining the relativistic transformation of the Gamow modes preserving the reality of the momentum.

We consider this construction as a quite natural one because from our point of view the Gamow modes are not independent entities but those contributions to the wave packet that obey purely exponential decay laws.

The novelty of our approach is that the vector $(z(\mathbf{k}), \mathbf{k})$ is not a four-vector, because the Lorentz transformation is not implementable in complex energy-momentum space. A representation of the Poincaré group including Gamow states can also be found in $[5,7]$.

In Section 2, we present the model and the exact solution obtained in [2]. In Section 3, we introduce decaying Gamow modes. In Section 4, we obtain the Lorentz transformation of exponentially decaying states. In Section 5, we present our conclusions.

\section{Exact results for the model of two interacting fields}

In this section, we present the results, which we obtained earlier in [2] for the local scalar boson field $\phi(x)$ interacting with a bilocal scalar boson field $\psi(x, q)$ in four-dimensional Minkowski space. The four-dimensional vector $x \equiv(t, \mathbf{x})$ has a space component represented by three-dimensional vector $\mathbf{x}$ and a time component $t$. The metrics of the Minkowski space is chosen $(+,-,-,-)$ such that in the units where $\hbar=c=1$ the scalar product is defined as

$$
\left(x, x^{\prime}\right) \equiv t t^{\prime}-\left(\mathbf{x}, \mathbf{x}^{\prime}\right)
$$

The local field is given by

$$
\phi(x)=\int \frac{d^{3} \mathbf{k}}{(2 \pi)^{3} 2 \omega(\mathbf{k})}\left[a^{\dagger}(\mathbf{k}) e^{i(\omega(\mathbf{k}) t-\mathbf{k x})}+a(\mathbf{k}) e^{-i(\omega(\mathbf{k}) t-\mathbf{k x})}\right],
$$

where $d^{3} \mathbf{k} /(2 \pi)^{3} 2 \omega(\mathbf{k})$ is relativistic invariant measure. The energy (frequency) of the field modes $\omega(\mathbf{k})$ is determined by the momentum $\mathbf{k}$ and the mass of the particle $\mathrm{M}$ according to the dispersion relation

$$
\omega(\mathbf{k})=\sqrt{\mathbf{k}^{2}+\mathrm{M}^{2}}
$$

The creation and annihilation operators of the field modes $a^{\dagger}(\mathbf{k})$ and $a(\mathbf{k})$ satisfy the boson commutation relations:

$$
\left[a(\mathbf{k}), a^{\dagger}\left(\mathbf{k}^{\prime}\right)\right]=(2 \pi)^{3} 2 \omega(\mathbf{k}) \delta^{3}\left(\mathbf{k}-\mathbf{k}^{\prime}\right) .
$$


The Hamiltonian of the free scalar field is

$$
H_{\mathrm{M}}=\int \frac{d^{3} \mathbf{k}}{(2 \pi)^{3} 2 \omega(\mathbf{k})} \omega(\mathbf{k}) a^{\dagger}(\mathbf{k}) a(\mathbf{k})
$$

The bilocal scalar field $\psi(x, q)$ has an additional internal degree of freedom $q$ which is one dimensional. We consider $\psi(x, q)$ satisfying the property $\psi(x, q)=\psi(x,-q)$. Then $\psi(x, q)$ is determined by the equation

$$
\left(\square-\mathrm{M}^{2}\right) \psi(x, q)=0, \quad \mathrm{M}^{2} \equiv 4 m^{2}-\frac{\partial^{2}}{\partial q^{2}},
$$

where $\square$ is the usual d'Alemlert operator and the quantum field $\psi(x, q)$ is given by

$$
\psi(x, q)=\int d^{3} \mathbf{k} \int_{E_{0}(\mathbf{k})}^{\infty} d E \frac{\cos (\kappa(E, \mathbf{k}) q)}{(2 \pi)^{4} \kappa(E, \mathbf{k})}\left[b^{\dagger}(E, \mathbf{k}) e^{i(\omega(\mathbf{k}) t-\mathbf{k x})}+b(E, \mathbf{k}) e^{-i(\omega(\mathbf{k}) t-\mathbf{k x})}\right] .
$$

The dispersion relation for $\psi(x, q)$ reads as

$$
\kappa(E, \mathbf{k})=\left(E^{2}-\mathbf{k}^{2}-4 m^{2}\right)^{1 / 2},
$$

where $m$ is the mass of the particle $\psi$. Therefore, the lower boundary of the integral over the energy $E$ in (2.7) is

$$
E_{0}(\mathbf{k})=\left(4 m^{2}+\mathbf{k}^{2}\right)^{1 / 2}
$$

The annihilation and creation operators of the field modes $b^{\dagger}(E, \mathbf{k})$ and $b(E, \mathbf{k})$ satisfy the boson commutation relation:

$$
\left[b(E, \mathbf{k}), b^{\dagger}\left(E^{\prime}, \mathbf{k}^{\prime}\right)\right]=(2 \pi)^{4} k(E, \mathbf{k}) \delta\left(E-E^{\prime}\right) \delta^{3}\left(\mathbf{k}-\mathbf{k}^{\prime}\right) .
$$

The Hamiltonian of the free bilocal field is

$$
H_{m}=\int d^{3} \mathbf{k} \int_{E_{0}(\mathbf{k})}^{\infty} \frac{d E}{(2 \pi)^{4} k(E, \mathbf{k})} E b^{\dagger}(E, \mathbf{k}), b(E, \mathbf{k}) .
$$

The commutation relations (2.4) and (2.10) induce an algebra of the Poincare group generators. Free fields $\phi(x), \psi(x, q)$ are scalars invariant with respect to the transformations of the Poincaré group. 
When we introduce interaction, we have to take care that the whole theory keeps this invariance. The simplest quadratic interaction, satisfying this requirement is (up to the coupling constant)

$$
V=-\int d^{3} \mathbf{x} \int_{-\infty}^{\infty} d q \psi(x, q) f(q) \phi(x)
$$

where $f(q)$ plays the role of the form factor introduced in order to avoid divergences. This is a scalar function with good asymptotic behavior, which will be specified below.

Then the Hamiltonian is the zero component of the four-momentum $[2,13]$

$$
P_{0}=H=H_{\mathrm{M}}+H_{m}+\lambda V
$$

where

$$
V=\int d^{3} \mathbf{k} \int_{E_{0}(\mathbf{k})}^{\infty} \frac{d E \alpha(k(E, \mathbf{k}))}{(2 \pi)^{3} 2 \omega(\mathbf{k}) k(E, \mathbf{k})}\left(a^{\dagger}(\mathbf{k})+a(-\mathbf{k})\right)\left(b^{\dagger}(E, \mathbf{k})+b(E,-\mathbf{k})\right)
$$

The generator of three-dimensional translations is

$$
\mathbf{P}=\int d^{3} \mathbf{k} \int_{E_{0}(\mathbf{k})}^{\infty} \frac{d E}{(2 \pi)^{4} k(E, \mathbf{k})} \mathbf{k} b^{\dagger}(E, \mathbf{k}) b(E, \mathbf{k})+\int \frac{d^{3} \mathbf{k}}{(2 \pi)^{3} 2 \omega(\mathbf{k})} \mathbf{k} a^{\dagger}(\mathbf{k}) a(\mathbf{k})
$$

The generators of three-dimensional rotations do not include interaction as usual:

$$
\begin{aligned}
J_{i j}= & -i \int d^{3} \mathbf{k} \int_{E_{0}(\mathbf{k})}^{\infty} \frac{d E}{(2 \pi)^{4} \kappa(E, \mathbf{k})} b^{\dagger}(E, \mathbf{k})\left(k_{j} \frac{\partial}{\partial k_{j}}-k_{j} \frac{\partial}{\partial k_{i}}\right) b(E, \mathbf{k}) \\
& -i \int \frac{d^{3} \mathbf{k}}{(2 \pi)^{3} 2 \omega(\mathbf{k})} a^{\dagger}(\mathbf{k})\left(k_{j} \frac{\partial}{\partial k_{j}}-k_{j} \frac{\partial}{\partial k_{i}}\right) a(\mathbf{k}),
\end{aligned}
$$

where indexes $i, j$ mark coordinates $k_{i}, k_{j}$ of three-dimensional vector $\mathbf{k}$. The Lorentz boost generators are

$$
\begin{aligned}
J_{0 i}= & i \int d^{3} \mathbf{k} \int_{E_{0}(\mathbf{k})}^{\infty} \frac{d E}{(2 \pi)^{4} \kappa(E, \mathbf{k})} b^{\dagger}(E, \mathbf{k})\left(E \frac{\partial}{\partial k_{j}}+k_{i} \frac{\partial}{\partial E}\right) b(E, \mathbf{k}) \\
& +i \int \frac{d^{3} \mathbf{k}}{(2 \pi)^{3} 2 \omega(\mathbf{k})} a^{\dagger}(\mathbf{k})\left(\omega(\mathbf{k}) \frac{\partial}{\partial k_{j}}\right) a(\mathbf{k}) \\
& +i \int d^{3} \mathbf{k} \int_{E_{0}(\mathbf{k})}^{\infty} \frac{d E}{(2 \pi)^{3} 2 \omega(\mathbf{k})} \frac{\lambda \alpha(\kappa(E, \mathbf{k}))}{\kappa(E, \mathbf{k})} \frac{1}{E}\left(E \frac{\partial}{\partial k_{j}}+k_{i} \frac{\partial}{\partial E}\right) \\
& \times\left[b(E, \mathbf{k})+b^{\dagger}(E,-\mathbf{k})\right]\left[a(-\mathbf{k})+a^{\dagger}(\mathbf{k})\right] .
\end{aligned}
$$


As we have shown in [2], when $\mathrm{M}>2 m$, the system becomes Poincaré nonintegrable. In this case, for $E \geq E_{0}(\mathbf{k})$, the solution of the eigenvalue problem

$$
\left[H, B^{\dagger}(E, \mathbf{k})\right]=E B^{\dagger}(E, \mathbf{k}) \quad[H, B(E, \mathbf{k})]=-E B(E, \mathbf{k})
$$

(where $[\cdot, \cdot]$ is a commutator) is given by the creation and annihilation operators $B_{\text {in }}^{\dagger}(E, \mathbf{k})$ and $B_{\text {in }}(E, \mathbf{k})$ :

$$
\begin{aligned}
\begin{array}{c}
B_{\text {in }}^{\dagger}(E, \mathbf{k})= \\
\text { out }
\end{array} & b^{\dagger}(E, \mathbf{k})+2 \pi \lambda \alpha(k(E, \mathbf{k})) G^{ \pm}(E, \mathbf{k}) \\
\times & \left\{\int_{E_{0}(\mathbf{k})}^{\infty} d E^{\prime} \frac{\lambda \alpha\left(k\left(E^{\prime}, \mathbf{k}\right)\right)}{k\left(E^{\prime}, \mathbf{k}\right)}\left(\frac{b^{\dagger}\left(E^{\prime}, \mathbf{k}\right)}{E^{\prime}-E \mp i 0}-\frac{b\left(E^{\prime},-\mathbf{k}\right)}{E^{\prime}+E}\right)\right. \\
& \left.-\frac{(E+\omega(\mathbf{k})) a^{\dagger}(\mathbf{k})+(E-\omega(\mathbf{k})) a(-\mathbf{k})}{2 \omega(\mathbf{k})}\right\}, \\
B_{\text {in }}(E, \mathbf{k})= & b(E, \mathbf{k})+2 \pi \lambda \alpha(k(E, \mathbf{k})) G^{\mp}(E, \mathbf{k}) \\
\times & \left\{\int_{E_{0}(\mathbf{k})}^{\infty} d E^{\prime} \frac{\lambda \alpha\left(k\left(E^{\prime}, \mathbf{k}\right)\right)}{k\left(E^{\prime}, \mathbf{k}\right)}\left(\frac{b\left(E^{\prime}, \mathbf{k}\right)}{E^{\prime}-E \pm i 0}-\frac{b^{\dagger}\left(E^{\prime},-\mathbf{k}\right)}{E^{\prime}+E}\right)\right. \\
& \left.-\frac{(E+\omega(\mathbf{k})) a(\mathbf{k})+(E-\omega(\mathbf{k})) a^{\dagger}(-\mathbf{k})}{2 \omega(\mathbf{k})}\right\} .
\end{aligned}
$$

The "in" and "out" solutions correspond to the boundary values $G^{ \pm}(E, \mathbf{k})=G(E \pm i 0, \mathbf{k})$ of the Green function

$$
G(z, \mathbf{k})=\left(\omega(\mathbf{k})^{2}-z^{2}-\int_{E_{0}(\mathbf{k})}^{\infty} d E^{\prime 2} \frac{2 \pi \lambda^{2} \alpha^{2}\left(k\left(E^{\prime}, \mathbf{k}\right)\right)}{k\left(E^{\prime}, \mathbf{k}\right)} \frac{1}{E^{\prime 2}-z^{2}}\right)^{-1}
$$

In the unstable case, which we discuss here, the Green function $G(z, \mathbf{k})$ is analytic in the $z$ complex plane except for the cut on the real line: $\left(-\infty,-E_{0}(\mathbf{k})\right] \cup\left[E_{0}(\mathbf{k}),+\infty\right)$. The jump on the cut is

$$
\begin{aligned}
& G^{+}(E, \mathbf{k})-G^{-}(E, \mathbf{k})=i \frac{4 \pi^{2} \lambda^{2} \alpha^{2}(k(E, \mathbf{k}))}{k(E, \mathbf{k})}|G(E, \mathbf{k})|^{2}, \quad E>E_{0}(\mathbf{k}), \\
& G^{+}(E, \mathbf{k})-G^{-}(E, \mathbf{k})=-i \frac{4 \pi^{2} \lambda^{2} \alpha^{2}(k(E, \mathbf{k}))}{k(E, \mathbf{k})}|G(E, \mathbf{k})|^{2}, \quad E<-E_{0}(\mathbf{k}),
\end{aligned}
$$

where $|G(E, \mathbf{k})|^{2} \equiv G(E+i 0, \mathbf{k}) G(E-i 0, \mathbf{k})$.

The Green function $G(E, \mathbf{k})$ satisfies the dispersion relation

$$
G(E, \mathbf{k})=\int_{E_{0}(k)}^{\infty} d E^{\prime 2} \frac{2 \pi \lambda^{2} \alpha^{2}\left(k\left(E^{\prime}, \mathbf{k}\right)\right)}{k\left(E^{\prime}, \mathbf{k}\right)} \frac{\left|G\left(E^{\prime}, \mathbf{k}\right)\right|^{2}}{E^{\prime 2}-E^{2}}
$$

which is the Källen-Lehman representation for the propagator [2]. 
The operators (2.19), (2.20) satisfy the boson commutation relations

$$
\begin{gathered}
{\left[B_{\text {in }}(E, \mathbf{k}), B_{\text {out }}^{\dagger}\left(E^{\prime}, \mathbf{k}^{\prime}\right)\right]=(2 \pi)^{4} \kappa(E, \mathbf{k}) \delta\left(E-E^{\prime}\right) \delta^{3}\left(\mathbf{k}-\mathbf{k}^{\prime}\right)} \\
{\left[B_{\text {out }}(E, \mathbf{k}), B_{\text {in }}^{\dagger}\left(E^{\prime}, \mathbf{k}^{\prime}\right)\right]=(2 \pi)^{4} \kappa(E, \mathbf{k}) \delta\left(E-E^{\prime}\right) \delta^{3}\left(\mathbf{k}-\mathbf{k}^{\prime}\right) \frac{G^{+}(E, \mathbf{k})}{G^{-}(E, \mathbf{k})}} \\
{\left[B_{\text {in }}(E, \mathbf{k}), B_{\text {out }}^{\dagger}\left(E^{\prime}, \mathbf{k}^{\prime}\right)\right]=(2 \pi)^{4} \kappa(E, \mathbf{k}) \delta\left(E-E^{\prime}\right) \delta^{3}\left(\mathbf{k}-\mathbf{k}^{\prime}\right) \frac{G^{-}(E, \mathbf{k})}{G^{+}(E, \mathbf{k})} .}
\end{gathered}
$$

The generators of the Poincare group are diagonal in the representation of $B_{\text {in }}^{\dagger}(E, \mathbf{k})$, $B_{\text {in }}(E, \mathbf{k})$ :

$$
P_{\mu}=\iint_{E_{0}(\mathbf{k})}^{\infty} \frac{d^{3} \mathbf{k} d E}{(2 \pi)^{4} \kappa(E, \mathbf{k})} k_{\mu} B_{\text {in }}^{\dagger}(E, \mathbf{k}) B_{\text {out }}(E, \mathbf{k}),
$$

where $P_{0}=H$ up to a constant, due to operator ordering, $k_{0}=\omega(\mathbf{k})$, and $\left(k_{1}, k_{2}, k_{3}\right)=\mathbf{k}$.

Three-dimensional rotations do not involve interaction terms (2.16) and therefore present no difficulties.

The commutation rules of the new operators with the Lorentz boosts are

$$
\left[J_{0 i}, B_{\text {out }}^{\dagger}(E, \mathbf{k})\right]=i\left(E \frac{\partial}{\partial k_{j}}+k_{i} \frac{\partial}{\partial k_{i}}\right) B_{\text {in }}^{\dagger}(E, \mathbf{k}) .
$$

In the original representation, the Fock space of states is spanned by the results of the action of the old operators on the bare vacuum state $|0\rangle$ defined as

$$
a(\mathbf{k})|0\rangle=b(E, \mathbf{k})|0\rangle=0, \quad \forall E, \mathbf{k} .
$$

However, due to virtual transitions, this is no more the vacuum state for the whole system because for the new operators, we have

$$
B_{\text {int }}(E, \mathbf{k})|0\rangle \neq 0
$$

The new relativistic invariant vacuum state $|\Omega\rangle$ satisfying

$$
B_{\text {in }}(E, \mathbf{k})|\Omega\rangle=0
$$

has the form

$$
|\Omega\rangle=C_{0} e^{V_{\text {vac }}}|0\rangle,
$$


where $C_{0}$ is the normalization factor. The transformation operator

$$
\begin{aligned}
V_{\mathrm{vac}}=\int & \frac{d^{3} \mathbf{k}}{(2 \pi)^{3} 2(\omega(\mathbf{k})+\xi(\mathbf{k}))} \\
& \times\left\{\int_{E_{0}(\mathbf{k})}^{\infty} d E \frac{\lambda \alpha(\kappa(E, \mathbf{k}))}{\kappa(E, \mathbf{k})} \gamma(E, \mathbf{k}) b^{\dagger}(E, \mathbf{k}) a^{\dagger}(-\mathbf{k})-\frac{\xi(\mathbf{k})}{2 \omega(\mathbf{k})} a^{\dagger}(\mathbf{k}) a^{\dagger}(-\mathbf{k})\right. \\
& +\int_{E_{0}(\mathbf{k})}^{\infty} d E d E^{\prime} \frac{\lambda^{2} \alpha(\kappa(E, \mathbf{k})) \alpha\left(\kappa\left(E^{\prime}, \mathbf{k}\right)\right)}{\kappa(E, \mathbf{k}) \kappa\left(E^{\prime}, \mathbf{k}\right)} \gamma(E, \mathbf{k}) \gamma\left(E^{\prime}, \mathbf{k}\right) \\
& \left.\times\left(\frac{1}{2}+\frac{\omega(\mathbf{k})+\xi(\mathbf{k})}{E+E^{\prime}}\right) b^{\dagger}(E, \mathbf{k}) b^{\dagger}(E,-\mathbf{k})\right\}
\end{aligned}
$$

is determined with the help of the factorization of the Green function [2]

$$
G(E, \mathbf{k})=\gamma(E, \mathbf{k}) \gamma(-E, \mathbf{k})
$$

The problem of the factorization of Green's functions often arises in theoretical and mathematical physics. In our case, as it was shown in [2], this is a well-posed problem provided by the asymptote of $G(E, \mathbf{k})$,

$$
G(E, \mathbf{k})=\frac{1}{\omega^{2}(\mathbf{k})-E^{2}}+O \frac{1}{E^{4}}
$$

The natural asymptotic condition is imposed on $\gamma(E, \mathbf{k})$,

$$
\gamma(E, \mathbf{k})^{-1}=-E-(\omega(\mathbf{k})+2 \xi(\mathbf{k}))+O \frac{1}{E}
$$

where $\xi(\mathbf{k})$ is a finite $\lambda^{2}$ correction to the term of the order $O(1)$ in the variable $E$. As the result $\gamma(E, \mathbf{k})$ has a cut $\left(-\infty ;-E_{0}(\mathbf{k})\right]$ and the following properties [2]:

$$
\begin{gathered}
\gamma(E, \mathbf{k})^{-1}+E+\omega(\mathbf{k})+2 \xi(\mathbf{k})+\int_{E_{0}(\mathbf{k})}^{\infty} d E^{\prime} \frac{2 \pi \lambda^{2} \alpha^{2}\left(\kappa\left(E^{\prime}, \mathbf{k}\right)\right) \gamma\left(E^{\prime}, \mathbf{k}\right)}{\kappa\left(E^{\prime}, \mathbf{k}\right)\left(E^{\prime}+E\right)}=0, \\
\int_{E_{0}(\mathbf{k})}^{\infty} d E^{\prime} \frac{2 \pi \lambda^{2} \alpha^{2}\left(\kappa\left(E^{\prime}, \mathbf{k}\right)\right)}{\kappa\left(E^{\prime}, \mathbf{k}\right)} \gamma\left(E^{\prime}, \mathbf{k}\right)=2 \xi(\mathbf{k})(\omega(\mathbf{k})+\xi(\mathbf{k})), \\
\left(E \frac{\partial}{\partial k_{j}}+k_{i} \frac{\partial}{\partial k_{i}}\right) \gamma(E, \mathbf{k})+\gamma(E, \mathbf{k}) \frac{\partial}{\partial k_{i}}(\omega(\mathbf{k})+\xi(\mathbf{k}))=0,
\end{gathered}
$$

the new creation operators $B_{\text {in }}(E, \mathbf{k})$ acting on the new vacuum state $|\Omega\rangle$ define new Fock spaces. The expression for the one-particle state in terms of the old operators may be obtained if we insert the explicit formulae for $B_{\text {in }}^{\dagger}(E, \mathbf{k})(2.19)$ and for $|\Omega\rangle$ (2.30) and (2.31) into

$$
\left|\Psi_{\text {in }}(E, \mathbf{k})\right\rangle=B_{\text {in }}^{\dagger}(E, \mathbf{k})|\Omega\rangle .
$$


Then using the commutation relations (2.4) and (2.10), we eliminate the old annihilation operators and obtain finally the expression for the one-particle eigenstate of the total Hamiltonian in the form

$$
\begin{aligned}
\left|\Psi_{\text {in }}(E, \mathbf{k})\right\rangle=\left\{b^{\dagger}(E, \mathbf{k})+\right. & \frac{\pi \lambda \alpha(\kappa(E, \mathbf{k}))}{\omega(\mathbf{k})+\xi(\mathbf{k})} \gamma(-E-i 0, \mathbf{k}) \\
& \times\left[\int_{E_{0}(\mathbf{k})}^{\infty} d E^{\prime} \frac{\lambda \alpha\left(\kappa\left(E^{\prime}, \mathbf{k}\right)\right)}{\kappa\left(E^{\prime}, \mathbf{k}\right)} \gamma\left(E^{\prime}, \mathbf{k}\right)\right. \\
& \left.\left.\times\left(1+\frac{2(\omega(\mathbf{k})+\xi(\mathbf{k}))}{E^{\prime}-E-i 0}\right) b^{\dagger}\left(E^{\prime}, \mathbf{k}\right)+a^{\dagger}(\mathbf{k})\right]\right\}|\Omega\rangle .
\end{aligned}
$$

Like in the nonrelativistic Friedrichs model $[1,3,10]$, the expression for the one-particle eigenstate of the total Hamiltonian contains the partial resolvent. Its role is now played by $\gamma(E, \mathbf{k})$, which has a cut on the positive semiaxes of the energy plane $[E(\mathbf{k}), \infty)$ and shares some of possible poles with the Green function $G(E, \mathbf{k})$.

\section{Decaying Gamow modes}

We first remark that in the stable case when $\mathrm{M}<2 m$, there exist additional solutions $A^{\dagger}(\mathbf{k}), A(\mathbf{k})$ of $(2.18)$ corresponding to the simple pole of $\gamma(-E, \mathbf{k})$ on the real line out of the cut. Together with the operators $B_{\text {in }}^{\dagger}(E, \mathbf{k}), B_{\text {in }}(E, \mathbf{k})$, they form a complete set in the whole space which is related to the original complete set of $a^{\dagger}(\mathbf{k}), a(\mathbf{k}), b^{\dagger}(E, \mathbf{k})$, and $b(E, \mathbf{k})$ through the Bogolubov transformation. This transformation establishes one-toone correspondence between the two complete sets.

In the unstable case, there is no pole of the Green function $G(z, \mathbf{k})$ in the physical sheet of the complex energy plane (the first Riemann sheet in the variable $z$ ). Therefore, the only solutions of $(2.18)$ are $B_{\text {in }}^{\dagger}(E, \mathbf{k})$ and $B_{\text {in }}(E, \mathbf{k})$ operators. Although the Bogolubov transformation relating the old and new operators exists as well, in this case, this transformation is no more unitary because there is no one-to-one correspondence between the old and new operators.

In the unstable case, the Green function $G(E, \mathbf{k})$ possesses poles in the second Riemann sheet of the complex plane. We denote by

$$
\mu_{c}^{2}=\mu^{2}-i \mu \Gamma
$$

the pole of $G(E, \mathbf{k})$ as a function of the Lorentz invariant variable $E^{2}-\mathbf{k}^{2}$ which is analytically continued through the cut from above in the right half-plane. As we will see below, $\mu_{c}$ may be considered as the complex mass of the Gamow state corresponding to this pole.

The corresponding poles of $G(E, \mathbf{k})$ as a function of $E$ analytically continued through the cut from above in the right half-plane and from below in the left half-plane are then $\pm z(\mathbf{k})$ such that

$$
z(\mathbf{k})=\left(\mathbf{k}^{2}+\mu_{c}^{2}\right)^{1 / 2}=\tilde{\omega}(\mathbf{k})-i \gamma(\mathbf{k}),
$$


where

$$
\begin{aligned}
& \tilde{\omega}(\mathbf{k})=\left\{\frac{\left[\left(\mathbf{k}^{2}+\mu^{2}\right)^{2}+\mu^{2} \Gamma^{2}\right]^{1 / 2}+\left(\mathbf{k}^{2}+\mu^{2}\right)}{2}\right\}^{1 / 2}, \\
& \gamma(\mathbf{k})=\left\{\frac{\left[\left(\mathbf{k}^{2}+\mu^{2}\right)^{2}+\mu^{2} \Gamma^{2}\right]^{1 / 2}-\left(\mathbf{k}^{2}+\mu^{2}\right)}{2}\right\}^{1 / 2}=\frac{\mu \Gamma}{2 \tilde{\omega}(\mathbf{k})} .
\end{aligned}
$$

We see that the imaginary part of the pole $\gamma(\mathbf{k})$, which is responsible for the life time of the field mode, depends now on the momentum $\mathbf{k}$.

For the weak coupling case, we can obtain from (3.4) and (2.21) the usually supposed expression

$$
\gamma(\mathbf{k})=\frac{\mathrm{M} \Gamma}{2 \omega(\mathbf{k})}
$$

which is valid only approximately up to the $\lambda^{4}$ terms.

In the vicinity of the pole $z(\mathbf{k})$, the Green function $G^{+}(E, \mathbf{k})$ has the following behaviour:

$$
G^{+}(E, \mathbf{k})=\gamma^{+}(E, \mathbf{k}) \gamma^{+}(-E, \mathbf{k})=\frac{R(\mathbf{k})}{\left(\mu_{c}^{2}-\mathbf{k}^{2}\right)-E^{2}}+\text { regular part }
$$

where $R(\mathbf{k})$ is the residue of $G(E, \mathbf{k})$ in the pole. Therefore the solution of the factorization problem in the vicinity of the pole has the form

$$
\begin{aligned}
\gamma^{+}(E, \mathbf{k}) & =-\frac{\sqrt{R(\mathbf{k})}}{E+\left(\mu_{c}^{2}+\mathbf{k}^{2}\right)^{1 / 2}}+\text { regular part }, \\
\gamma^{+}(-E, \mathbf{k}) & =\frac{\sqrt{R(\mathbf{k})}}{E-\left(\mu_{c}^{2}+\mathbf{k}^{2}\right)^{1 / 2}}+\text { regular part. }
\end{aligned}
$$

Using this fact, we can write (2.37) as

$$
\left|\Psi_{\text {in }}(E, \mathbf{k})\right\rangle=\frac{1}{E-z(\mathbf{k})}\left|\Phi^{\mathrm{G}}(E, \mathbf{k})\right\rangle+\text { regular part }
$$

where $\left|\Phi^{\mathrm{G}}(E, \mathbf{k})\right\rangle$ is the Gamow vector associated with the resonance pole $z(\mathbf{k})$. These vectors acquire meaning in the extended space where the Gamow kets are considered as distributions acting on the test functions represented by the bra vectors

$$
\langle\psi|=\int d^{3} \mathbf{k} \int_{E_{0}(\mathbf{k})}^{\infty} \frac{d E}{(2 \pi)^{4} \mathcal{\kappa}(E, \mathbf{k})} \psi(E, \mathbf{k})\langle\Omega| B_{\text {out }}(E, \mathbf{k}) .
$$

Using commutation relations (2.4) and (2.10), we compute the bracket of (2.37) and (3.9) as

$$
\left\langle\psi \mid \Psi_{\text {in }}(E, \mathbf{k})\right\rangle=\psi(E, \mathbf{k}) \frac{\gamma^{+}(-E)}{\gamma^{-}(-E)} .
$$


Note that the bra vectors (3.9) are chosen as the wave packets of the bra modes determined by the operator $B_{\text {out }}(E, \mathbf{k})$ including the function $G^{+}(E, \mathbf{k})$, which may be analytically continued through the cut from above as it is necessary for calculations of the pole contribution. As a result, in (3.10) the function $\gamma^{+}(-E)$ being analytically continued through the cut to the lower half-plane has a pole at $z(\mathbf{k})$ and the analytic continuation of $\gamma^{-}(-E)$ to the lower half-plane has neither singularity nor zero at this point. Then restricting the variety of admissible functions $\psi(E, \mathbf{k})$ to a set of appropriate functions with good properties, we see that $\left\langle\psi(E, \mathbf{k}) \mid \Psi_{\text {in }}(E, \mathbf{k})\right\rangle$ admits a meromorphic extension to the lower half-plane having a pole at $z(\mathbf{k})$. Extracting the pole contribution from (3.10), we define

$$
\psi(z(\mathbf{k}), \mathbf{k}) \frac{\sqrt{R(\mathbf{k})}}{\gamma^{-}(-z(\mathbf{k}), \mathbf{k})} \equiv C(\mathbf{k})\left\langle\psi \mid \Phi^{\mathrm{G}}(\mathbf{k})\right\rangle
$$

where $\left|\Phi^{\mathrm{G}}(\mathbf{k})\right\rangle$ is the Gamow state and $C(\mathbf{k})$ is the normalization constant. Using the fact that $\left|\Psi_{\text {in }}(E, \mathbf{k})\right\rangle$ is an eigenstate of the total Hamiltonian

$$
P_{0}\left|\Psi_{\text {in }}(E, \mathbf{k})\right\rangle=E\left|\Psi_{\text {in }}(E, \mathbf{k})\right\rangle
$$

we conclude that the Gamow state is also an eigenstate of the total Hamiltonian with the complex eigenvalue $[2,4,6]$

$$
P_{0}\left|\Phi^{\mathrm{G}}(\mathbf{k})\right\rangle=z(\mathbf{k})\left|\Phi^{\mathrm{G}}(\mathbf{k})\right\rangle .
$$

Then for the time evolution of the Gamow state, we have the expression

$$
e^{-i t P_{0}}\left|\Phi^{\mathrm{G}}(\mathbf{k})\right\rangle=e^{-i z(\mathbf{k}) t}\left|\Phi^{\mathrm{G}}(\mathbf{k})\right\rangle=e^{-i \tilde{\omega}(\mathbf{k}) t} e^{-\gamma(\mathbf{k}) t}\left|\Phi^{\mathrm{G}}(\mathbf{k})\right\rangle,
$$

which shows the exponential decay of the Gamow mode.

\section{Lorentz transformation of decaying states}

We here describe how the Lorentz boosts transform the exponentially decaying states. Our point of view is based on the fact that in scattering experiments, in the laboratory frame, one observes the wave packets with real momenta. Therefore the Lorentz transformation of the Gamow state should not change the reality of the momentum. This is, of course, not so if $(z(\mathbf{k}), \mathbf{k})$ is a four-vector. Therefore, we take the point of view that the Lorentz boosts act only on the wave packets without pointwise implementation, that is,

$$
U(\alpha)\left|\Phi^{\mathrm{G}}\right\rangle=U(\alpha)\left|\Phi^{\mathrm{G}}(z(\mathbf{k}), \mathbf{k})\right\rangle \neq\left|\Phi^{\mathrm{G}}\left(U^{-1}(\alpha)(z(\mathbf{k}), \mathbf{k})\right)\right\rangle .
$$

We will now construct $U_{\alpha}$. In order to isolate the exponentially decaying state, one has to perform an analytic extension to the complex energy plane nearby the maximum on the real line where we expect to find a resonance. We find in this way the contribution of the Gamow modes into the wave packet. The same procedure applies for the observers in moving frames. Observing the same wave packet, they have another curve on the real line transformed from the one in the laboratory frame by the appropriate Lorentz boost. The 
momentum of this packet is real again. Then by analytic extension in the complex energy plane, one finds the representation of the transformed wave packet through the Gamow modes. It is the transformation from the Gamow modes giving major contribution to the wave packet in the laboratory frame "at rest" to the Gamow modes contributing to the wave packet in the "moving frame" that we identify as the Lorentz transformation of exponentially decaying states.

We start with the transformation of the eigenstates of the Hamiltonian. Using the commutation relation of the new operators with the Lorentz boost and the Lorentz invariance of the vacuum state, we have for the eigenstate of the total Hamiltonian $\left|\Psi_{\text {in }}(E, \mathbf{k})\right\rangle(2.37)$

$$
J_{o, i}\left|\Psi_{\text {in }}(E, \mathbf{k})\right\rangle=i\left(E \frac{\partial}{\partial k_{j}}+k_{i} \frac{\partial}{\partial k_{i}}\right)\left|\Psi_{\text {in }}(E, \mathbf{k})\right\rangle
$$

Then the Lorentz transformation generated by (4.2) is

$$
U(\alpha)=\exp \left\{i \alpha_{i} J_{0 i}\right\}
$$

where $\alpha_{i}$ is a vector with the components

$$
\alpha_{i}=\alpha n_{i}, \quad \mathbf{n}^{2}=1, i=1,2,3,
$$

that acts on $\left|\Psi_{\text {in }}(E, \mathbf{k})\right\rangle$ as

$$
U(\alpha)\left|\Psi_{\text {in }}(E, \mathbf{k})\right\rangle=\left|\Psi_{\text {in }}\left(E_{\alpha}^{\prime}, \mathbf{k}_{\alpha}^{\prime}\right)\right\rangle
$$

where

$$
\begin{aligned}
& E_{\alpha}^{\prime}=E \cosh \alpha-(\mathbf{n k}) \sinh \alpha, \\
& \mathbf{k}_{\alpha}^{\prime}=\mathbf{k}-\mathbf{n}((\mathbf{k n}) \cosh \alpha-E \sinh \alpha)
\end{aligned}
$$

Extracting the pole contribution (in the complex E plane) from both parts of (4.2), we come to the formal expression for the Gamow state

$$
U(\alpha)\left|\Phi^{\mathrm{G}}(\mathbf{k})\right\rangle=\left|\Phi^{\mathrm{G}}\left(\mathbf{k}_{\alpha}^{\prime}\right)\right\rangle
$$

where

$$
\mathbf{k}_{\alpha}^{\prime}=\mathbf{k}-\mathbf{n}((\mathbf{k n}) \cosh \alpha-z(\mathbf{k}) \sinh \alpha)
$$

This leads to the complex momentum $\mathbf{k}_{\alpha}^{\prime}$ of the transformed state. The complex value of the momentum is not admissible from the physical point of view. Therefore, this procedure is not justified. In addition to the strange meaning of the complex momentum, another natural question arises. In the reference frame in which we have obtained the Gamow state $\left|\Phi^{\mathrm{G}}(\mathbf{k})\right\rangle$ as the pole contribution $\left|\Psi_{\text {in }}(E, \mathbf{k})\right\rangle$, the momentum $\mathbf{k}$ is real. The same state from the point of view of any observer moving in this reference frame will have complex momentum. Why the reference frame in which we obtained the Gamow vector first is so exceptional? 
We suggest to overcome this difficulty by applying the Lorentz boost not to the Gamow state but to the original eigenstate of the full energy-momentum four-vector, from which the Gamow state was obtained. Such transformation of the original state in the Hilbert space corresponds to the change of the reference frame form the initial one to the reference frame of the moving observer. In the new reference frame, the state $\left|\Psi_{\text {in }}(E, \mathbf{k})\right\rangle$ will have different energy and momentum due to the Lorentz boost. The energy and momentum of the transformed state $\left|\Psi_{\text {in }}\left(E^{\prime}, \mathbf{k}^{\prime}\right)\right\rangle$ are obtained according to (4.6). The Gamow state $\left|\Phi^{\mathrm{G}}(\mathbf{k})\right\rangle$ in the initial reference frame is obtained by analytic continuation in the variable $E$ and the transformed Gamow state $\left|\Phi^{\mathrm{G}}\left(\mathbf{k}^{\prime}\right)\right\rangle$, that is, the same state but in the moving reference frame, is obtained by analytic continuation of $\left|\Psi\left(E^{\prime}, \mathbf{k}^{\prime}\right)\right\rangle$ in the variable $E^{\prime}$ for the fixed value $\mathbf{k}^{\prime}$.

This procedure, however, has an ambiguity, because when we analytically continue $\left|\Psi_{\text {in }}(E, \mathbf{k})\right\rangle$, we do not fix $E$ as it is the variable, as we use for the analytic continuation. But in order to fix $\mathbf{k}^{\prime}$ after the Lorentz transformation of $\left|\Psi_{\text {in }}(E, \mathbf{k})\right\rangle$, we have to know both $E$ and $\mathbf{k}$ in the initial state, which is not the case as we do not fix $E$.

We avoid this apparent ambiguity using the Gamow wave packets, which we define as follows. We first form a one-particle wave packet from the energy-momentum eigenstates in the Hilbert space

$$
|f\rangle=\int d^{3} \mathbf{k} \int_{E_{0}(\mathbf{k})}^{\infty} \frac{d E}{(2 \pi)^{4} \kappa(E, \mathbf{k})} f(E, \mathbf{k})\left|\Psi_{\text {in }}(E, \mathbf{k})\right\rangle,
$$

where the function $f(E, \mathbf{k})$ determines the shape of the wave packet. When we describe a particle with energy $E_{0}$ and the momentum $\mathbf{k}_{0}$, we consider a wave packet localized in the momentum representation around the values $E_{0}$ and $\mathbf{k}_{0}$. It is the function $f(E, \mathbf{k})$ that determines this localization giving appropriate "weight" to the field modes $\left|\Psi_{\text {in }}(E, \mathbf{k})\right\rangle$, thus "shaping" the wave packet around $E_{0}$ and $\mathbf{k}_{0}$.

Extracting the pole contribution from the integrand of (4.9), we obtain the Gamow wave packet

$$
\left|f^{\mathrm{G}}\right\rangle=\int d^{3} \mathbf{k} \frac{f(z(\mathbf{k}), \mathbf{k})}{(2 \pi)^{4} \kappa(z(\mathbf{k}), \mathbf{k})}\left|\Phi^{\mathrm{G}}(\mathbf{k})\right\rangle .
$$

The state of the Gamow wave packet is represented now by complex function $f(z(\mathbf{k}), \mathbf{k})$. However, it still shapes the wave packet around some real values of the momentum $\mathbf{k}_{0}^{\mathrm{G}}$ in the same way as $f(E, \mathbf{k})$.

After the extraction of the pole contribution

$$
\left|f^{\mathbf{b g}}\right\rangle=|f\rangle-\left|f^{\mathrm{G}}\right\rangle
$$

we have the remaining part, which is usually called "background" and can be written as

$$
\left|f^{\mathrm{bg}}\right\rangle=\int d^{3} \mathbf{k} \int_{C_{z(\mathbf{k})}} \frac{d E}{(2 \pi)^{4} \kappa(E, \mathbf{k})} f(E, \mathbf{k})\left|\Psi_{\text {in }}(E, \mathbf{k})\right\rangle
$$


where the contour of the integration runs along the real line from $E_{0}(\mathbf{k})$ above the cut as in (4.9) but then on the way it goes to the second Riemann sheet below the cut of $\gamma(-E-$ $i 0, \mathbf{k})$, which is present in $\left|\Psi_{\text {in }}(E, \mathbf{k})\right\rangle$. It makes a loop around the pole $z(\mathbf{k})$, returns to the first sheet, and continues to $\infty$ along the real line above the cut. Although the background gives negligible contribution to physically relevant expectation values in the exponential era of the decay, it has to be taken into account in the discussion of the representations of the Poincaré group.

In order to obtain the Lorentz transformation of the Gamow wave packet, we start, as we have suggested, with the transformation of the initial wave packet $|f\rangle$ in the Hilbert space. This replaces $E$ and $\mathbf{k}$ in the variables in $\left|\Psi_{\text {in }}(E, \mathbf{k})\right\rangle$ in (4.9) by $E^{\prime}, \mathbf{k}^{\prime}$, which are given by (4.6),

$$
U(\alpha)|f\rangle=\int d^{3} \mathbf{k} \int_{E_{0}(\mathbf{k})}^{\infty} \frac{d E}{(2 \pi)^{4} \kappa(E, \mathbf{k})} f(E, \mathbf{k})\left|\Psi_{\text {in }}\left(E^{\prime}, \mathbf{k}^{\prime}\right)\right\rangle
$$

In order to see the shape of the transformed wave packet, we change the variables in (4.13) by the transformation, which is inverse to (4.6), and obtain

$$
E=E\left(E^{\prime}, \mathbf{k}^{\prime}\right), \quad \mathbf{k}=\mathbf{k}\left(E^{\prime}, \mathbf{k}^{\prime}\right)
$$

Then using the properties of the Lorentz invariant measure in (4.13), we come to

$$
U(\alpha)|f\rangle=\int d^{3} \mathbf{k}^{\prime} \int_{E_{0}\left(\mathbf{k}^{\prime}\right)}^{\infty} d E^{\prime} \frac{f\left(E\left(E^{\prime}, \mathbf{k}^{\prime}\right), \mathbf{k}\left(E^{\prime}, \mathbf{k}^{\prime}\right)\right)}{(2 \pi)^{4} \kappa\left(E^{\prime}, \mathbf{k}^{\prime}\right)}\left|\Psi_{\text {in }}\left(E^{\prime}, \mathbf{k}^{\prime}\right)\right\rangle
$$

The shape of the transformed wave packet is determined by the new function $f\left(E\left(E^{\prime}, \mathbf{k}^{\prime}\right)\right.$, $\left.\mathbf{k}\left(E^{\prime}, \mathbf{k}^{\prime}\right)\right)$. This function shapes the wave packet around other values $E_{0}^{\prime}$ and $\mathbf{k}_{0}^{\prime}$. Then we extract the pole contribution from (4.15) and obtain

$$
U(\alpha)\left|f^{\mathrm{G}}\right\rangle=\int d^{3} \mathbf{k}^{\prime} \frac{f\left(E\left(z\left(\mathbf{k}^{\prime}\right), \mathbf{k}^{\prime}\right), \mathbf{k}\left(z\left(\mathbf{k}^{\prime}\right), \mathbf{k}^{\prime}\right)\right)}{(2 \pi)^{4} \kappa\left(z\left(\mathbf{k}^{\prime}\right), \mathbf{k}^{\prime}\right)}\left|\Phi^{\mathrm{G}}\left(\mathbf{k}^{\prime}\right)\right\rangle .
$$

This gives the transformed Gamow wave packet, which will be shaped around the other fixed value $\mathbf{k}_{0}^{\mathrm{G}^{\prime}}$. The remaining part gives the transformed background

$$
\begin{aligned}
U(\alpha)\left|f^{\mathrm{bg}}\right\rangle & =U(\alpha)|f\rangle-U(\alpha)\left|f^{\mathrm{G}}\right\rangle \\
& =\int d^{3} \mathbf{k} \int_{C_{z\left(\mathbf{k}^{\prime}\right)}} d E^{\prime} \frac{f\left(E\left(E^{\prime}, \mathbf{k}^{\prime}\right), \mathbf{k}\left(E^{\prime}, \mathbf{k}^{\prime}\right)\right)}{(2 \pi)^{4} \kappa\left(E^{\prime}, \mathbf{k}^{\prime}\right)} f(E, \mathbf{k})\left|\Psi_{\text {in }}\left(E^{\prime}, \mathbf{k}^{\prime}\right)\right\rangle
\end{aligned}
$$

Thus, we have constructed a new nonpointwise representation of the Poincare group. This representation includes exponentially decaying Gamow states. The obtained transformation of the complex Gamow wave packet leads to a new wave packet which is again a combination of the Gamow states with real momentum. 


\section{Conclusion}

We have shown that relativistic transformation of the Gamow modes, which obey strictly exponential decay law, can be introduced in a consistent way with the help of decaying wave packets observed in experiments. This transformation keeps the momentum of Gamow modes real. Due to the physical requirement of the reality of the momentum, $(z(\mathbf{k}), \mathbf{k})$ cannot be a four-vector.

We know that the existence of Gamow states manifests intrinsic irreversibility of dynamical systems [3]. Therefore, by construction of the consistent Lorentz transformation for Gamow modes as a part of new representation of the Poincare group, we show the compatibility of the notion of irreversibility with relativistic theory. Another representation of the Poincaré group including Gamow states can also be found in $[5,7]$.

\section{Acknowledgments}

The authors acknowledge the financial support of the European Commission Grant HPHA-CT-2001-40002. The nonpointwise implementable action of the Lorentz boost (4.16) has been the original intuitive remark of Ilya Prigogine, which we discussed with him shortly before his passing away. Therefore, we feel obliged to include his name as a coauthor. On this occasion we would like to express our gratitude for many years of exciting scientific interaction and friendship. E. Karpov is grateful to the fund of Professor Prigogine for the support of his research.

\section{References}

[1] I. Antoniou, M. Gadella, E. Karpov, I. Prigogine, and G. Pronko, Gamov algebras, Chaos Solitons Fractals 12 (2001), no. 14-15, 2757-2775.

[2] I. Antoniou, M. Gadella, I. Prigogine, and G. Pronko, Relativistic Gamow vectors, J. Math. Phys. 39 (1998), no. 6, 2995-3018.

[3] I. Antoniou and I. Prigogine, Intrinsic irreversibility and integrability of dynamics, Phys. A 192 (1993), no. 3, 443-464.

[4] A. Bohm and M. Gadella, Dirac kets, Gamow Vectors and Gelfand Triplets, Lecture Notes in Physics, vol. 348, Springer-Verlag, Berlin, 1989.

[5] A. Bohm, H. Kaldass, S. Wickramasekara, and P. Kielanowski, Relativistic Gamow vectors II, preprint, 2000, http://arXiv.org/abs/hep-th/9905213.

[6] A. R. Bohm, Time asymmetry and quantum theory of resonances and decay, Internat. J. Theoret. Phys. 42 (2003), no. 10, 2317-2338.

[7] A. R. Bohm and M. J. Mithaiwala, Relativistic resonances, semigroup representation of Poincaré transformations, the exponential decay law and deviations thereof, J. Phys. A 35 (2002), no. 40, 8479-8503.

[8] P. Davis, That mysterious flow, Scientific American (2002), 40-47.

[9] E. Karpov, G. Ordonez, T. Petrosky, and I. Prigogine, Quantum transitions in interacting fields, Phys. Rev. A 66 (2002), no. 1, 1-17.

[10] E. Karpov, I. Prigogine, T. Petrosky, and G. Pronko, Friedrichs model with virtual transitions. Exact solution and indirect spectroscopy, J. Math. Phys. 41 (2000), no. 1, 118-131.

[11] G. Ordonez, T. Petrosky, and I. Prigogine, Quantum transitions and dressed unstable states, Phys. Rev. A 63 (2001), no. 5, 1-23.

[12] T. Petrosky, G. Ordonez, and I. Prigogine, Space-time formulation of quantum transitions, Phys. Rev. A 64 (2001), no. 6, 1-21. 
[13] S. S. Schweber, An Introduction to Relativistic Quantum Field Theory, Row, Peterson and Company, New York, 1961, Foreword by Hans A. Bethe.

I. Antoniou: Department of Mathematics, Aristotle University of Thessaloniki, 54124 Thessaloniki, Greece

E-mail address: iantonio@math.auth.gr

E. Karpov: Chaos and Innovation Research Unit, Department of Mathematics, Aristotle University of Thessaloniki, 54124 Thessaloniki, Greece

Current address: International Solvay Institutes for Physics and Chemistry, ULB Campus Plaine, CP 231, Boulevard du Triomphe, Brussels 1050, Belgium

E-mail address: ekarpov@ull.ac.le

I. Prigogine: International Solvay Institutes for Physics and Chemistry, ULB Campus Plaine, CP 231, Boulevard du Triomphe, Brussels 1050, Belgium; Center for Studies in Statistical Mechanics and Complex Systems, The University of Texas at Austin, Austin, Texas 78712, USA

G. Pronko: Institute for High Energy Physics, 142284 Protvino, Moscow, Russia

Current address: Chaos and Innovation Research Unit, Department of Mathematics, Aristotle University of Thessaloniki, 54124 Thessaloniki, Greece

E-mail address: pronko@mx.ihep.su 


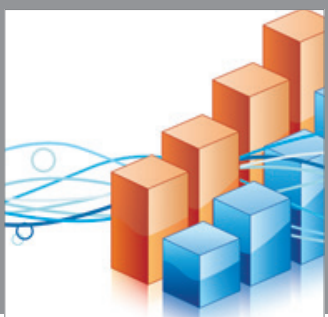

Advances in

Operations Research

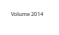

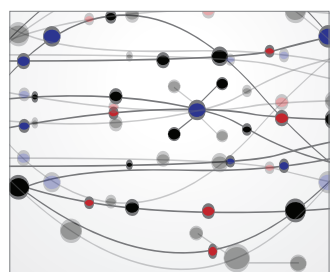

\section{The Scientific} World Journal
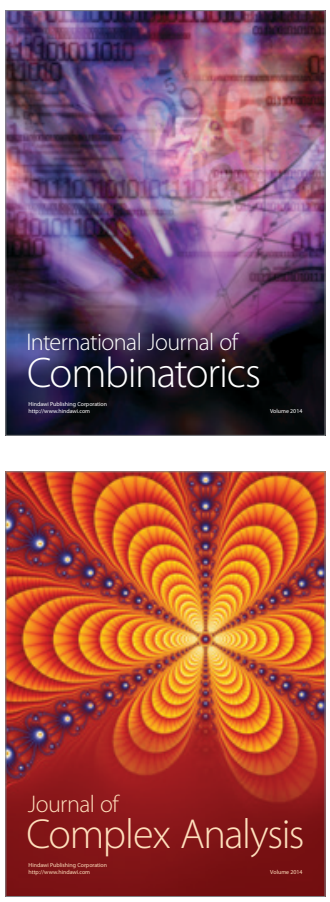

International Journal of

Mathematics and

Mathematical

Sciences
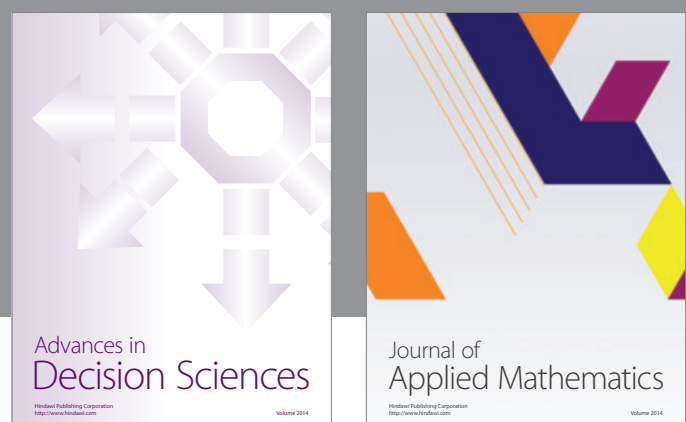

Journal of

Applied Mathematics
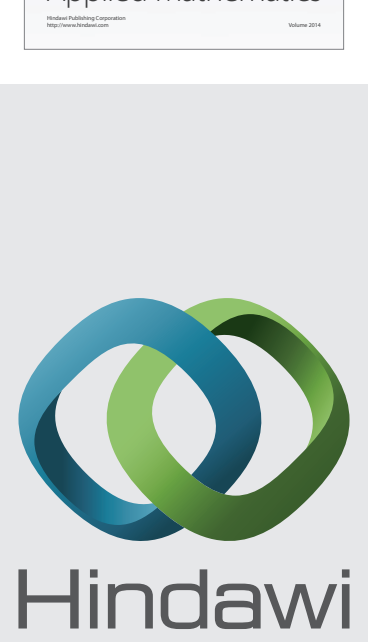

Submit your manuscripts at http://www.hindawi.com
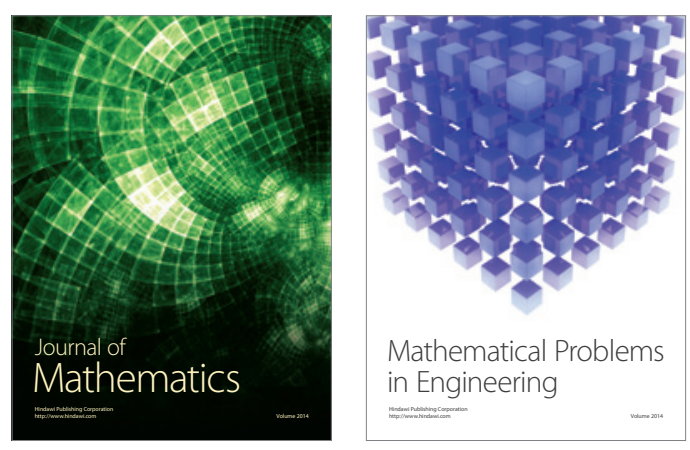

Mathematical Problems in Engineering
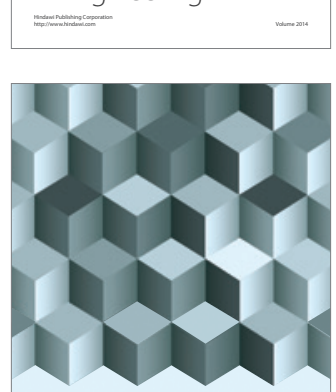

Journal of

Function Spaces
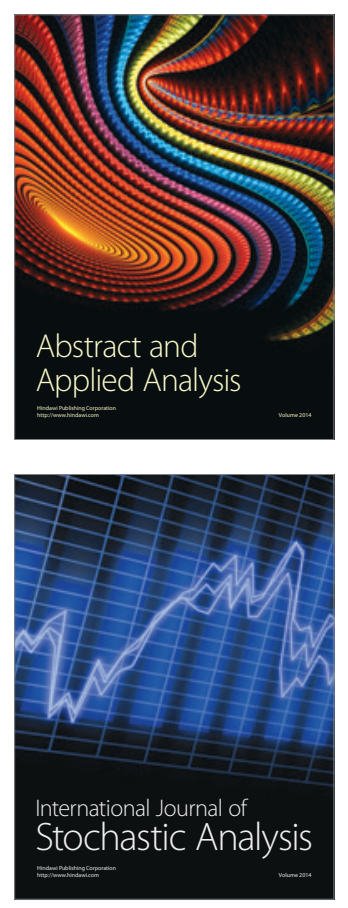

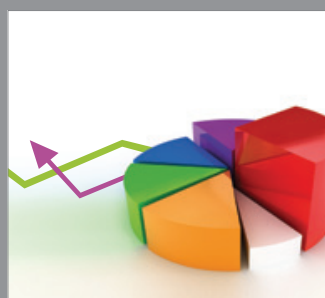

ournal of

Probability and Statistics

Promensencen
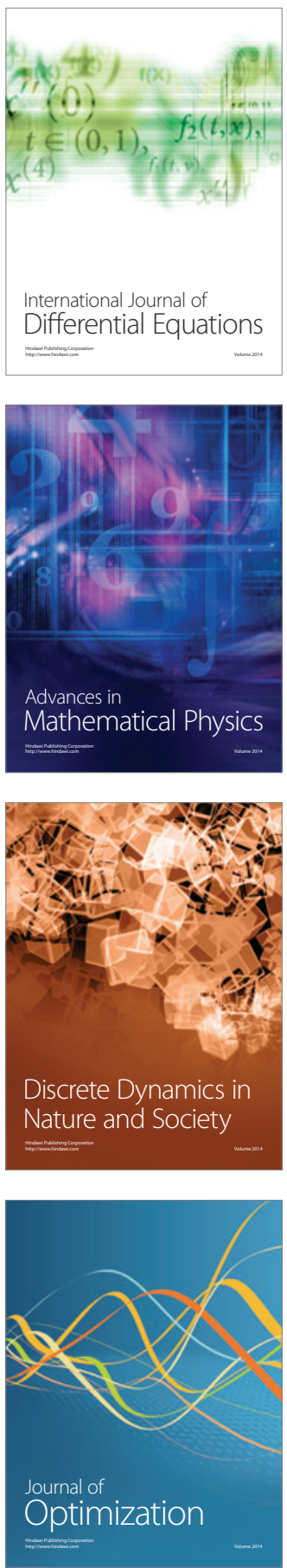